\title{
MedienPädagogik
}

Zeitschrift für Theorie und Praxis der Medienbildung

Themenheft Nr. 36: Teilhabe in einer durch digitale Medien geprägten Welt Perspektiven des wissenschaftlichen Nachwuchses

Herausgegeben von Tim Riplinger, Jan Hellriegel und Ricarda Bolten

\section{Interdisziplinäre Entwicklung eines Vernetzungsmoduls «Medienpädagogik»}

\section{Ein Praxisbericht zur Lehramtsausbildung an der Technischen Universität Darmstadt}

Marcella Haller, Franco Rau, Sophie Schaper und Naheela Ulrich

\begin{abstract}
Zusammenfassung
Angehende Lehrerinnen und Lehrer stehen in einer von digitalen Medien durchdrungenen Welt vor der Herausforderung, digital gestützte Lehr-Lernsituationen zu gestalten und den Erwerb von digitalen Kompetenzen ihrer Schülerinnen und Schüler zu fördern. Um diese Herausforderung professionell bearbeiten zu können, wird die Entwicklung einer medienpädagogischen Kompetenz im Lehramtsstudium als Voraussetzung betrachtet. Dafür bedarfes angemessener Angebote der Bildungs- und Fachwissenschaften sowie der Fachdidaktiken. Mit dem vorliegenden Praxisbericht wird ein Einblick in die Netzwerkarbeit und Konzeption des Vernetzungsmoduls Medienpädagogik im Lehramtsstudium an der Technischen Universität Darmstadt gegeben. Es wird aufgezeigt, wie eine für die Zusammenarbeit entwickelte Vernetzungsmatrix zur Entwicklung des Moduls Medienpädagogik und zur Verbindung unterschiedlicher disziplinärer Perspektiven beitragen konnte. Die Matrix unterstützte als Orientierungshilfe die Zusammenarbeit von Akteurinnen und Akteuren unterschiedlicher Disziplinen. Zum einen ermöglichte sie eine strukturierte und transparente Konzeption des Moduls Medienpädagogik. Zum anderen diente sie als Rahmen zur Entwicklung interdisziplinärer Lehrprojekte.
\end{abstract}

Interdisciplinary development of a module «Medienpädagogik»

\begin{abstract}
In a world permeated by digital media, prospective teachers face the challenge of designing digitally-supported teaching and learning and promoting the acquisition of students' digital literacy. To handle this challenge professionally, the development of media literacy in the teacher training program is considered as a prerequisite. This requires appropriate courses in the fields of basic science, specialised/specialist science and subject-related didactics. This practical report provides an insight into the conception of the module Medienpädagogik in teacher training at the Technische Universität Darmstadt. For that reason, we developed an instrument to collaborate with lecturers from different
\end{abstract}


disciplines. The article shows that the instrument can support collaboration of lecturers with different disciplinary perspectives. On the one hand, it enabled a structured and transparent conception of the module Medienpädagogik. On the other hand, it served as a framework for the development of interdisciplinary teaching projects.

Vernetzung als Chance für die Förderung medienpädagogischer Kompetenz im Lehramtsstudium

Die medienpädagogische Kompetenz von Lehrerinnen und Lehrern wird als Voraussetzung betrachtet, um Angebote zur Medienbildung in der Schule entwickeln und realisieren zu können (Tulodziecki 2012). Um zum Aufbau professioneller Handlungsfähigkeit von zukünftigen Lehrpersonen beizutragen, bedarf es im Lehramtsstudium angemessener Angebote der Bildungs- und Fachwissenschaften sowie der Fachdidaktiken. Eine Vernetzung der verschiedenen in der Lehramtsausbildung aktiven Akteurinnen und Akteure mit der Medienpädagogik betrachten wir in diesem Zusammenhang als notwendig, um eine fächerintegrative Förderung einer medienpädagogischen Kompetenz zu ermöglichen. Aus diesem Grund wurde im Rahmen des Projekts «MINTplus: systematischer und vernetzter Kompetenzaufbau in der Lehrerbildung» an der Technischen Universität Darmstadt ein neues Vernetzungsmodul Medienpädagogik entwickelt. Als ein im sogenannten Vernetzungsbereich verankertes Modul ist die Zusammenarbeit der verschiedenen Akteurinnen und Akteure bei der Konzeption des Moduls Medienpädagogik zentral. Um in den Vernetzungstreffen einen strukturierten und zielführenden Austausch zu unterstützen, wurde eine Vernetzungsmatrix (kurz Matrix) entwickelt, die vorgängige Kernüberlegungen des Arbeitsbereichs angemessen kommunizieren kann.

Ziel dieses Beitrags ist es, einen Überblick über die Entwicklung des neuen Moduls Medienpädagogik in Kooperation mit Fachdidaktikerinnen und Fachdidaktikern zu geben. Zunächst werden bildungspolitische Rahmenbedingungen zur Verankerung von Medienbildung in der Lehramtsausbildung aufgezeigt und die Rahmenbedingungen der Modulentwicklung benannt. Anschliessend wird das Vorgehen inklusive der Vernetzungstreffen und der verwendeten Matrix beschrieben. Im folgenden Kapitel werden die Ergebnisse dieser Gespräche und das entwickelte Veranstaltungskonzept erläutert. Zuletzt werden aktuelle Kooperationsprojekte beschrieben und ein Ausblick auf die Fortführung des Projekts gegeben.

\section{Medienbildung im Lehramtsstudium}

Medienbildung wird in zahlreichen bildungspolitischen Dokumenten als relevanter staatlicher Erziehungs- und Bildungsauftrag formuliert (z.B. BMBF 2010; LKM 2015; KMK 2017). Die Beschreibung von Medienkompetenz als neue Kulturtechnik (z.B. 
KMK 2012; LKM 2015) unterstreicht die Relevanz von Medienbildung in bildungspolitischen und -administrativen Kontexten. Der Begriff «Medienbildung» lässt sich in diesem Zusammenhang als «breitbandiges Konzept» zur pädagogisch strukturierten Medienkompetenzvermittlung verstehen (Jörissen 2011, 214). Dieses Begriffsverständnis zeigt sich sowohl in Betrachtung älterer Veröffentlichungen wie dem Expertenbericht BMBF (2010), als auch jüngeren bildungspolitischen und administrativen Dokumenten. Im Positionspapier der Länderkonferenz Medienbildung (LKM 2015) wird Medienkompetenz zur «unverzichtbaren Kulturtechnik» erklärt und deren Erwerb soll «durch eine verbindliche, grundlegende und systematische schulische Medienbildung» (LKM 2015, 3) erfolgen. Im Koalitionsantrag des Bundestags (24.03.2015) werden neben dem Medienkompetenzbegriff auch die Formulierungen «digitale Bildung» (Bundestag 24.03.2015, 3 f.) und «digitale Medienkompetenz» verwendet (Bundestag 24.03.2015, 5). Die institutionelle Vermittlung von Medienkompetenz wird in bildungspolitischen sowie medienpädagogischen Debatten unter den Formulierungen einer «integrativen Medienbildung» (z.B. Kammerl und Atzeroth 2013; Aßmann und Herzig 2015), einer «integrativen Aufgabe» (z.B. BMBF 2010) sowie als integrativer Ansatz in den Unterrichtsfächern (z.B. Arbeitsgruppe Erziehungswissenschaft 1997; KMK 2017) diskutiert. Der Begriff der Integration soll in diesem Zusammenhang zum Ausdruck bringen, dass «neue Medien für die einzelnen Unterrichtsfächer eine grosse Bedeutsamkeit haben und zur Veränderung von fachbezogenen Lehr- und Lernprozessen beitragen» (Arbeitsgruppe Erziehungswissenschaft 1997, 45).

Um den komplexen Anforderungen zur Medienbildung in der Schule gerecht zu werden, bedarf es einer angemessenen Vorbereitung und Begleitung von Lehrerinnen und Lehrern in allen Phasen der Lehrerinnen- und Lehrerbildung. Umso überraschender erscheint es, dass, im Gegensatz zu den vielfältigen Leitbildern für die schulische Medienbildung zum Projektbeginn, kaum vergleichbare Leitbilder für die Lehrerinnen- und Lehrerbildung identifiziert werden konnten. Zudem sind die existierenden bildungspolitischen und -administrativen Dokumente zur Medienbildung in der Lehrerinnen- und Lehrerbildung durchaus kritisch zu bewerten. Dass entsprechend zukünftige Lehrerinnen und Lehrer gemäss der skizzierten Leitbilder angemessen darauf vorbereitet werden, Medienbildung im Rahmen ihrer Schulfächer integrativ vermitteln zu können, darf in Anlehnung an die Positionen von Kammerl und Ostermann (2010), Kammerl (2015), Niesyto (2012) und Moser (2012) begründet angezweifelt werden. Kammerl und Ostermann $(2010,48)$ beschreiben in ihrer «Expertise zum Stellenwert der Medienkompetenzförderung in Schulen» den «Teufelskreislauf fehlender Medienbildung». Die Autorin und der Autoren markieren u.a. das Problem, dass Lehramtsstudierende zum Studienbeginn kaum über Medienkompetenzen verfügen und «die meisten von ihnen erfahren», so Kammerl und Ostermann $(2010,48)$, «keine oder kaum Ausbildung in diesem Bereich». Zu einem ähnlichen 
Schluss kommt Kammerl auch fünf Jahre später: «Medienkompetenzförderung als zentrale Aufgabe schulischer Medienbildung ist nicht im Bewusstsein aller (zukünftigen) Lehrpersonen verankert» (Kammerl 2015, 33). Zugleich sind medienpädagogische Angebote im Lehramtsstudium, wie Kammerl $(2015,33)$ exemplarisch für die Christian-Albrechts-Universität zu Kiel und die Universität Hamburg zeigt, zwar belegbar, aber nicht verpflichtend. Gründe für die unzureichenden Kompetenzen der Lehramtsstudierenden sind für Kammerl und Ostermann $(2010,48)$ u.a. die eigenen Schulerfahrungen bzw. das Problem, dass Medienbildung in Schulen nicht adäquat realisiert wird. Zugleich reproduzieren jene unvorbereiteten Lehramtsabsolventinnen und -absolventen das Problem fehlender Medienbildung an Schulen, wenn sie über keine angemessene medienpädagogische Kompetenz verfügen.

Ein Ausweg aus diesem Teufelskreis sehen Kammerl und Ostermann (2010,49) in einer «stärkeren Verankerung in den Lehrplänen» sowie einer «Stärkung der Medienbildung in der Lehrerbildung». Diese Problematik und eine vergleichbare Lösungsperspektive einer medienpädagogischen Lehrerinnen- und Lehrerbildung diskutiert Niesyto (2012) für das Bundesland Baden-Württemberg. Auch Moser $(2012,267)$ plädiert dafür, dass die «Medienbildung in der Ausbildung der Lehrkräfte aller Schulstufen massiv zu verstärken ist». Die notwendige Verstärkung begründet sich für Moser $(2012,267)$ auch aufgrund der aktuellen Situation, dass eine Medienbildung für Lehrerinnen und Lehrer an Hochschulen «nur mit schwachen Anteilen vertreten [ist]». Schliesslich plädiert auch die Sektion Medienpädagogik der Deutschen Gesellschaft für Erziehungswissenschaft $(2017,6)$ für die Entwicklung medienpädagogischer Module. Anknüpfend an diese Gestaltungsperspektiven erfolgte die Entwicklung und Umsetzung des verpflichtenden Vernetzungsmoduls Medienpädagogik an der TU Darmstadt.

Projekt MINTplus: systematischer und vernetzter Kompetenzaufbau in der Lehrerbildung - Rahmenbedingungen

Das Projekt «MINTplus: systematischer und vernetzter Kompetenzaufbau in der Lehrerbildung» der Technischen Universität Darmstadt wird im Rahmen der «Qualitätsoffensive Lehrerbildung» gefördert. Eine erste Förderphase des Projekts lief von Juli 2015 bis Dezember 2018. Das Projekt umfasste Teilprojekte zur Profilierung und Optimierung der Strukturen der Lehrerinnen und Lehrerbildung, Qualitätsverbesserung des Praxisbezugs, Verbesserung der professionsbezogenen Beratung, Fortentwicklung der Lehrerbildung bzgl. Heterogenität und Inklusion sowie Fortentwicklung von Fachlichkeit, Didaktik und Bildungswissenschaften (Bruder und Bachmann 2017, 6). Zentrales Ergebnis dieser Förderphase ist die Weiterentwicklung des Studiengangs Lehramt am Gymnasium (LaG), welcher mit einer neuen Studienordnung im Wintersemester 2017/18 startete. Es wurde ein Vernetzungsbereich als neues 
Studienelement geplant, welcher die Förderung fächerübergreifender Kompetenzen der Studierenden ermöglichen soll. Mit einem Umfang von insgesamt 20 LP umfasst der Vernetzungsbereich vier Studienmodule, die inhaltlich und methodisch aufeinander abgestimmt sind. In diesem Rahmen wurde das für alle LaG-Studierenden verpflichtende Modul Medienpädagogik entwickelt.

Das neue Pflichtmodul Medienpädagogik umfasst insgesamt 5 CP. Die Inhalte werden wie folgt im Modulhandbuch beschrieben:

«Das Modul zielt auf den Aufbau professioneller pädagogischer Handlungskompetenz von Lehrkräften in einer von digitalen Medien geprägten Kultur. Die wissensbasierte Auseinandersetzung mit dem Wandel der Bildung durch digitale Medien wird durch gestaltungs- und transferorientierte Elemente ergänzt.» (Modulhandbuch 2017, 7).

Als Qualifikationsziele für das Modul wird dort benannt:

«Die Studierenden ...

- können Veränderungen in einer durch Informations- und Kommunikationstechnologien durchdrungenen Welt erkennen und deren Bedeutung für Erziehungs- und Bildungsprozesse verstehen.

- können den Einsatz von (digitalen) Medien pädagogisch und didaktisch begründen und argumentativ vertreten.

- können Einsatzbereiche für Neue Medien in Bildungsprozessen hinsichtlich ihrer lern- und bildungsförderlichen Potenziale differenzieren und beurteilen.

- können den fachspezifischen Einsatz von (digitalen) Medien an Beispielen aus den Studienfächern mediendidaktisch analysieren und beurteilen.

- können medial unterstützte Lehr-Lernarrangements so planen und gestalten, dass neue Möglichkeiten der Veranschaulichung, der Verständnisförderung sowie des selbstständigen und kooperativen Arbeitens erfahrbar werden.» (Modulhandbuch 2017, 7).

Das Modul soll idealerweise im 5. Semester des Lehramtstudiums belegt werden und in einem Semester abschliessbar sein. Die Modulkonzeption erfolgte im Austausch mit Akteurinnen und Akteuren aus den Fachdidaktiken in Form von Vernetzungsgesprächen gemäss des Anspruchs, ein interdisziplinäres Vernetzungsmodul zu entwickeln, das an die Inhalte des Vernetzungsbereichs sowie der Fachdidaktik anschlussfähig sein soll. 
Interdisziplinäre Entwicklung in Form von Vernetzungstreffen

Für die Modulentwicklung war die Vernetzung eine wesentliche Aufgabe. Im Zeitraum von 2016 bis 2018 fanden zwei Runden von Vernetzungstreffen statt. Diese dienten dem fachübergreifenden und fächerverbindenden Austausch zwischen den Lehrenden aus dem Bereich der Medienpädagogik und den Professorinnen und Professoren, wie auch wissenschaftlichen Mitarbeiterinnen und Mitarbeitern der im Lehramt an Gymnasien (LaG) vertretenen Fachdidaktiken (Sport, Deutsch, Geschichte, Ethik/ Philosophie, Physik, Mathematik, Informatik, Chemie, Biologie). Ziel war zunächst, die Akteurinnen und Akteure der Fachdidaktik über relevante medienpädagogische Themen und Veranstaltungen zu informieren und darüber hinaus die medienpädagogischen Anteile innerhalb der Fachdidaktiken systematisch zu erfassen. Im Vergleich der Angebote der Medienpädagogik und der Fachdidaktiken konnten Überschneidungen, Dopplungen und Lücken identifiziert werden. Durch die gemeinsame Arbeit im Rahmen des Projekts "Qualitätsoffensive Lehrerbildung» konnten Kontakte zu den jeweiligen Akteurinnen und Akteuren hergestellt werden.

Die Vernetzungstreffen mit den teilnehmenden Fachdidaktikerinnen und Fachdidaktikern wurden entsprechend individuell vereinbart und gestaltet. Die ersten Vernetzungstreffen fanden von Mitte 2016 bis Mitte 2017 statt und dienten dem inhaltlichen Austausch (z.B. über Methoden, Workshop-Angebote, verwendete medientechnische Infrastruktur). Um inhaltliche Anschlussstellen zu erweitern und die Ergebnisse zu systematisieren folgte Ende 2017 bis Anfang 2018 eine zweite Runde.

\section{Methode zur Verständigung - Vernetzungsmatrix}

Zur Strukturierung der Vernetzungsarbeit wurde eine Matrix als Arbeits- und Gesprächsgrundlage konzipiert (vgl. Abb. 1). Diese umfasst in Bezug auf zukünftige Lehrerinnen und Lehrer und deren medienpädagogische Kompetenz drei Bereiche:

1. Bildungsraum Schule in einer von digitalen Medien durchdrungenen Welt/Kultur umfasst die Perspektive, dass angehende Lehrerinnen und Lehrer sich des Einflusses digitaler Medien auf die gesellschaftliche und schulische Entwicklung bewusst werden sollen. Darüber hinaus sollen sie in der Lage sein, diese kritisch zu reflektieren, um somit verantwortungsvoll mit digitalen Technologien in digitalen Räumen zu handeln. Die Formulierung dieses Aspektes erfolgte in Anlehnung an die Kompetenzbereiche «die Wahrnehmung von medienbezogenen Erziehungsund Bildungsaufgaben» sowie «die Entwicklung medienpädagogischer Konzepte in der Schule» (Tulodziecki 2012, 282).

2. Unterricht mit digitalen Medien planen, durchführen und reflektieren beschreibt, dass die angehenden Lehrerinnen und Lehrer dazu befähigt werden sollen digitale Medien «systematisch und selbstverständlich» (Moser 2010, 34) in ihre Unterrichtspraxis einzubeziehen. Dieser Bereich ist anschlussfähig an das Konzept 
der mediendidaktischen Kompetenz (Mayrberger 2012; Blömecke 2005) sowie an den Kompetenzbereich der «Mediennutzung zur Anregung und Unterstützung von Lernprozessen» (Tulodziecki 2012, 282).

3. Individuelle Medienkompetenz beschreibt in Anlehnung an Tulodziecki $(1997,116)$ die «Fähigkeit und Bereitschaft zu einem sachgerechten, selbstbestimmten, kreativen und sozial verantwortlichen Handeln» in einer digital geprägten Welt. Die individuelle Medienkompetenz von Lehramtsstudierenden wird als Voraussetzung betrachtet, eine medienpädagogische Kompetenz entwickeln zu können.

\begin{tabular}{|l|l|l|l|}
\hline & $\begin{array}{c}\text { Bildungsraum Schule } \\
\text { in einer digital ge- } \\
\text { prägten Welt/Kultur }\end{array}$ & $\begin{array}{c}\text { Unterricht mit digita- } \\
\text { len Medien planen \& } \\
\text { reflektieren }\end{array}$ & $\begin{array}{c}\text { Individuelle Medien- } \\
\text { kompetenz }\end{array}$ \\
\hline Wissen & & & \\
\hline Können & & & \\
\hline Haltung & & & \\
\hline
\end{tabular}

Abb. 1.: Matrix als Orientierungshilfe zur Durchführung von Vernetzungstreffen (Eigene Darstellung).

In Anlehnung an Moser (2010) erfolgt mit der Matrix zudem eine Unterscheidung von (a) Wissen, (b) Können und (c) Haltung. Dabei umfasst (a) Wissen diejenigen Kenntnisse und Theorien, über die eine Person verfügt. (b) Können, welches von Moser als «wissensbasiertes Handeln» beschrieben wird, zeichnet sich dadurch aus, dass die Person in der Lage ist, ihr Wissen und ihre Fähigkeiten selbstständig in konkrete Handlungen umzusetzen (Moser 2010, 29). Als Voraussetzung für den Erwerb von Wissen und dessen Umsetzung gilt eine von Bereitschaft gekennzeichnete (c) Haltung der Person. Für diese Kategorisierung wurde sich entschieden, da sie als gut zugänglich für die einzelnen Akteurinnen und Akteure bewertet wurde und die Dreiteilung in «Wissen - Können - Haltung» an weitere Konzepte anschlussfähig ist (z.B. DGfE Sektion Medienpädagogik 2017; Kleiner und Klenk 2017).

\section{Durchführung und Nachbereitung der Vernetzungstreffen}

Zum Einstieg in die Vernetzungsgespräche wurden die Matrix und damit verbundene Ziele zunächst erläutert, sowie offene Fragen geklärt. Um die Matrix als Arbeitsgrundlage nutzen zu können wurde sie visualisiert und zur Strukturierung sowie zur Dokumentation des Gespräches verwendet. Im Fokus standen zwei Leitfragen:

- Status Quo: Welche Inhalte/Themen/Werkzeuge werden von der Fachdidaktik in ihren Lehrveranstaltungen bereits abgedeckt/genutzt?

- Wünsche: Welche Wünsche in Form von Inhalten/Werkzeugen haben die Fachdidaktikerinnen und Fachdidaktiker an das Modul Medienpädagogik? 
Nach dem Gespräch wurden die Ergebnisse in einer digitalen Matrix dokumentiert und mit den Ergebnissen aus anderen Vernetzungstreffen zusammengeführt. Zur Auswertung wurden diese im ersten Schritt in zwei Matrizen aufgeteilt. Matrix Eins umfasste alle Beiträge zur ersten Leitfrage (Status Quo) und die Zweite entsprechend die der zweiten Leitfrage (Wünsche). Daraufhin wurden die Beiträge in einem zweiten Schritt nach Themenschwerpunkten und Überschneidungen geclustert, um eine quantitative Auswertung zu ermöglichen. Im dritten Schritt wurde versucht, diese mit den bereits vorhandenen medienpädagogischen Modulinhalten in Verbindung zu bringen. Hierfür wurden die Interessen und Perspektiven der Fachdidaktiken mit den eigenen Zieldimensionen aus medienpädagogischer Perspektive abgeglichen. Als Auswertungsgrundlage dienten die zwei Matrizen und die gebildeten Cluster, welche reflektiert und entsprechend zugeordnet wurden. Die unterschiedlichen Cluster und die dadurch entstandenen Interessensmuster ermöglichten eine Orientierung von konkreten bis zu übergreifenden Inhalten und Werkzeugen für das Modul Medienpädagogik.

\section{Ergebnisse}

Im Folgenden werden ausgewählte Ergebnisse des Prozesses vorgestellt. Es wird zwischen den inhaltlichen Erkenntnissen aus den Vernetzungstreffen und einer Diskussion der entwickelten Vernetzungsmatrix als Verständigungshilfe unterschieden. Zudem wird eine Skizze des aktuellen Veranstaltungskonzeptes des Vernetzungsmoduls Medienpädagogik vorgestellt.

\section{Inhaltliche Ergebnisse der Vernetzungstreffen}

Die Ergebnisse der Vernetzungstreffen wurden mit Hilfe der Matrix für die zwei zentralen Fragestellungen ausgewertet. Im Prozess erfolgte eine Unterscheidung von zwei Fächergruppen: MINT steht für Mathematik, Informatik, Naturwissenschaften und Technik GESS steht in diesem Verfahren für Gesellschafts- und Geschichtswissenschaften sowie Sport- und Sprachwissenschaften. Zur Übersichtlichkeit werden in den folgenden Darstellungen Aussagen von Akteurinnen und Akteuren der unterschiedlichen Fachdidaktiken mit jeweils unterschiedlichen Farben gekennzeichnet (Abb. 2 und 3). Die MINT-Fächer umfassen an der Technischen Universität Darmstadt Mathematik (blau), Informatik (pink), Physik (braun), Chemie (gelb) und Biologie (grün). Zu den GESS-Fächern gehören Geschichte (orange), Ethik/Philosophie (türkis), Sport (rot) und Deutsch (lila). Die dargestellten Punkte symbolisieren jeweils Aussagen zu konkreten Inhalten und Werkzeugen und es erfolgt eine Verortung und Diskussion innerhalb der Vernetzungsmatrix. 
In der ersten Matrix wird ein Überblick über den aktuellen Status Quo gegeben (Abb. 2). Sichtbar wird, dass ein deutlicher Schwerpunkt auf dem Kompetenzbereich (2) Unterricht mit digitalen Medien planen, durchführen und reflektieren liegt. Die Verteilung auf die drei Kategorien Wissen, Können und Haltung ist relativ ausgeglichen. Die fachdidaktischen Akteurinnen und Akteure sprechen häufig von Medien als Mittel zur Gestaltung von Unterricht. Seltener werden Medien als Unterrichtsinhalt bzw. -gegenstand diskutiert. Digitalisierungs- und Mediatisierungsprozesse auf struktureller Ebene wurden in den Gesprächen nicht explizit thematisiert. Darüber hinaus wurde von verschiedenen Fachdidaktikerinnen und Fachdidaktikern erwähnt, dass sie den Inhaltsbereich (1) Bildungsraum Schule in einer von digitalen Medien durchdrungenen Welt/Kultur stärker der Medienpädagogik zuordnen und sich selbst im Inhaltsbereich (2) Unterricht mit digitalen Medien planen, durchführen und reflektieren, verorten. Andere wiederum verankern sich in jedem Inhaltsbereich und jeder Kategorie. Auffällig war, dass die MINT-Fächer tendenziell mehr Medieneinsatz und -bezug, als die GESS-Fächer artikulierten.

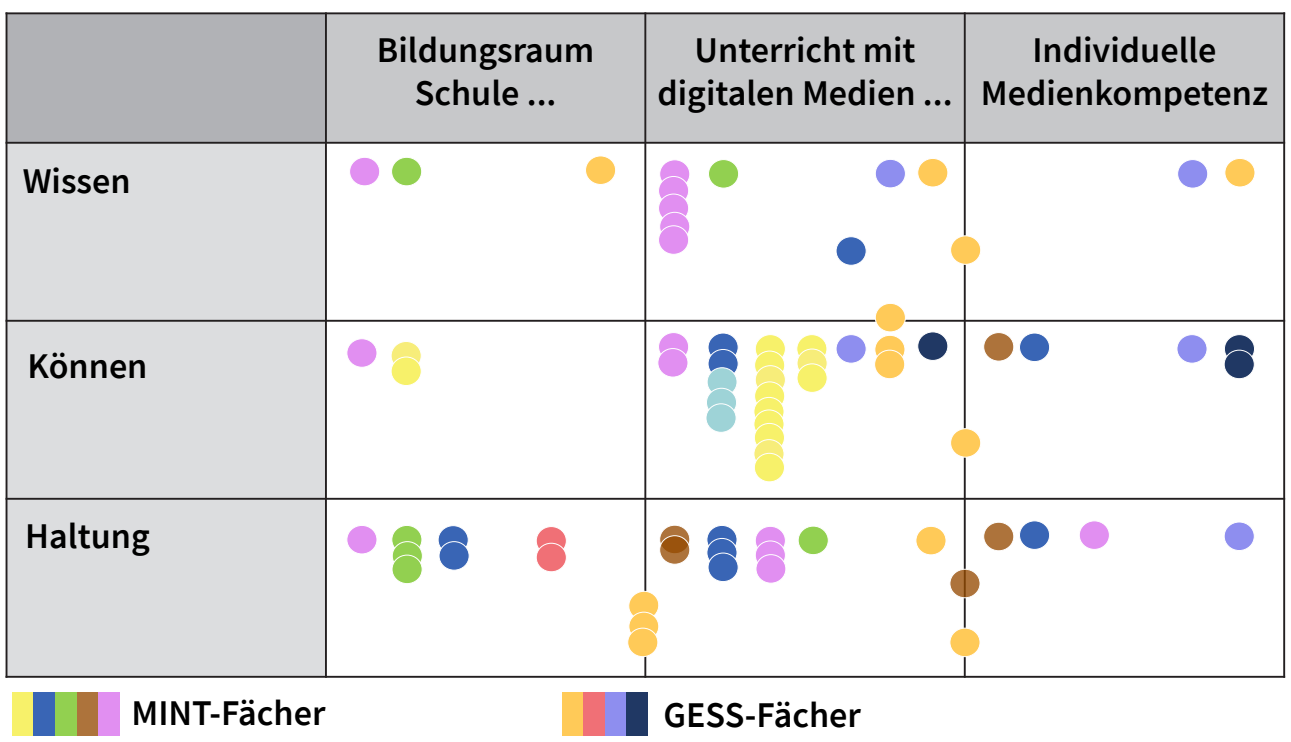

Abb. 2.: Übersicht über die quantitative Auszählung von Aussagen zum Status Quo (Eigene Darstellung).

In der zweiten Matrix zu den Wünschen der Fachdidaktikerinnen und Fachdidaktiker an das Modul Medienpädagogik (Abb. 3) liegen die Schwerpunkte insgesamt stärker auf den Bereichen (1) Bildungsraum Schule in einer von digitalen Medien durchdrungenen Welt/Kultur und (3) Individuelle Medienkompetenz. Es wird erkennbar, dass Fachdidaktikerinnen und Fachdidaktiker einen Bedarf zur Vermittlung medienpädagogischer Kompetenzen über die Werkzeug-Ebene hinaus sehen. Zugleich wird die Vermittlung entsprechender Kompetenzen jedoch nicht als genuine Aufgabe 
der Fachdisziplinen verstanden. Schwerpunkte liegen zudem auf den Kategorien (a) «Wissen» und (b) «Können». Der Unterschied zwischen MINT- und GESS-Fächer wiederholt sich in der zweiten Matrix.

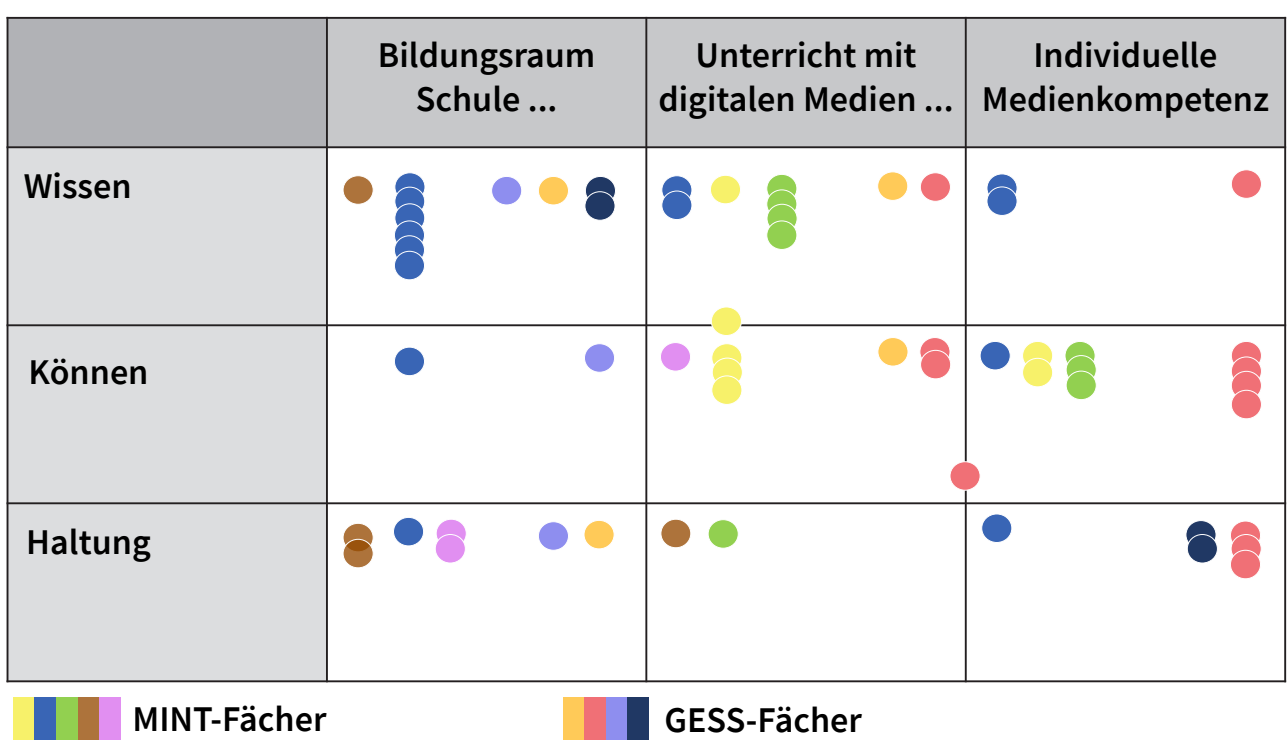

Abb. 3.: Übersicht über die quantitative Auszählung von Aussagen zu den Wünschen und Erwartungen an das Modul Medienpädagogik (Eigene Darstellung).

Aus den Ergebnissen lassen sich hilfreiche Hinweise für die Konzeption und Durchführung des Moduls Medienpädagogik identifizieren. Zum Beispiel verwenden fast alle Fachdidaktiken sowohl fachspezifische als auch allgemeine digitale Werkzeuge und erwarten dies auch von dem Vernetzungsmodul. Exemplarische Aussagen umfassten allgemeine Tools (z.B. «Moodle», «Mahara», «Wikis»), fachspezifische Werkzeuge (z.B. «Diagnosesoftware», «Tabellenkalkulation») sowie digitale Endgeräte (z.B. «Tablets» und «Interaktive Whiteboards»). Zudem wird gewünscht, den Fokus des Moduls auf die aktive Gestaltung und Entwicklung von Unterrichtsmedien und Medienprojekten zu legen (z.B. «Videos in der Lehre», «Podcasts», «Medien gestalten»).

Von Seiten der Lehrenden und Lernenden wurde eine offene und reflexive Haltung als relevant betrachtet. Dies beinhaltet die Aneignung und Reflexion der eigenen Medienkompetenz (z.B. «technisches Enabling», «kritisch-reflektierend») sowie eine selbstverständliche Nutzung digitaler Medien in der Hochschullehre (z.B. «demonstrative Mediennutzung»). Darüber hinaus wurden konkrete (Wunsch-)Themen genannt (z.B. «digitale Spiele», «Medienkritik», «Jugendmedienschutz»), welche die Lehrenden im Modul Medienpädagogik verorten. 
Vernetzungsmatrix als Verständigungshilfe und Kooperationsanlass

Der Einsatz der Matrix als Verständigungsinstrument ist hilfreich. Die Unterscheidung der Kompetenzbereiche sowie die Differenzierung zwischen Wissen, Können und Haltung eröffnete zahlreiche Anknüpfungs- und Diskussionspunkte für die Fachdidaktikerinnen und Fachdidaktiker. Ausserdem hilft die Matrix dabei aufzuzeigen, dass Medienpädagogik mehr als Unterricht mit Medien ist. Die Reaktionen der Fachdidaktikerinnen und Fachdidaktiker bestätigten dies. In den Vernetzungsgesprächen sollte daher deutlich hervorgehoben werden, dass der fächerverbindende Austausch auf Augenhöhe im Mittelpunkt steht und nicht das Abfragen bzw. Kontrollieren von medienpädagogischen Inhalten («Die Medienpolizei»). Aktuell wurden die stark disziplinär geprägten Perspektiven der Akteurinnen und Akteure als Herausforderung empfunden. In den Gesprächen dominierte etwa die Medienintegration auf Werkzeug-Ebene. Weiterhin bestand das vordergründige Interesse der Akteurinnen und Akteure teilweise in Bezug auf die Behandlung von Inhalten oder Werkzeugen, die ausschliesslich für ihre Disziplin relevant sind. Darüber hinaus sollte die Konzeption des Moduls den heterogenen Erfahrungen und Wünschen der Akteurinnen und Akteure gerecht werden (z.B. MINT- und GESS-Fächer). Damit ging oftmals eine Erwartungshaltung einher, die das Vernetzungsmodul als medienpädagogisches «Dienstleistungsangebot» auffasste, was lediglich dazu nütze, die gewünschten Inhalte zu vermitteln, ohne eigene Inhalte einzubringen. Mit Hilfe der Matrix konnten derartige Probleme nicht überwunden werden, allerdings ermöglichte sie, solche zu identifizieren.

Zudem lässt sich festhalten, dass die Fachdidaktikerinnen und Fachdidaktiker nach dem Gespräch auf der Basis der Ergebnisse Kooperationsmöglichkeiten erkannt haben und die Bereitschaft zur Umsetzung dieser sehr hoch war. Es wurden bereits Projekte mit der Fachdidaktik Geschichte, Chemie und Ethik/Philosophie durchgeführt. Diese sollen in Zukunft intensiviert werden. Darüber hinaus sind weitere Kooperationen mit der Fachdidaktik Informatik und der Physik in Planung. Die Kooperationsprojekte sind vielfältig und an die unterschiedlichen Bedürfnisse der Fachdidaktikerinnen und Fachdidaktikern angepasst: Gemeinsam mit der Fachdidaktik Chemie wurden Seminarsitzungen zum Thema Datenmanagement und Creative Commons Lizenzen entworfen. Die Medienpädagogik nahm dabei eine beratende Rolle bzgl. didaktischer und gestalterischer Fragen ein. Ein weiteres Kooperationsprojekt mit der Fachdidaktik Ethik/Philosophie zum Thema «Neosokratische Gespräche im Digitalen Zeitalter» umfasst die gemeinsame Produktion eines Lehr-Lern-Videos und die Durchführung von Workshops, in denen medienpädagogisch relevante Fragestellungen mit Hilfe neosokratischer Gesprächstechniken erörtert werden. 


\section{Lehrveranstaltungskonzept des Moduls Medienpädagogik}

Das mit Unterstützung der Erfahrungen und Auswertungen der Vernetzungsgespräche entwickelte Lehrveranstaltungskonzept (vgl. Abb. 4) umfasst drei wesentliche Bestandteile: (1.) einem Selbststudium mit einem festgelegtem Materialkorpus, (2.) einer Lehrveranstaltung sowie (3.) einer Projektarbeit. Der Materialkorpus muss innerhalb des ersten Drittels der Laufzeit im Selbststudium (ca. 60h) studiert werden. Er besteht aus einer Reihe von Vorlesungsaufzeichnungen sowie Grundlagen- und Vertiefungsliteratur. Der Baustein wird mit einer Abgabe via ePortfolio (Mahara) beendet. Das Seminar kann aus einer Reihe von Veranstaltungen mit unterschiedlichen Schwerpunkten gewählt werden. Aufbauend auf die Auseinandersetzungen mit dem Materialkorpus werden spezifische Inhalte vertieft und die Projektarbeit betreut. In der Projektarbeit (60h) entwerfen die Studierenden in Kleingruppenarbeit ein Medienprodukt (z.B. Video, Podcast, Poster) und formulieren gemeinsam eine ergänzende schriftliche Begründung (Hausarbeit). Medienprodukt und Hausarbeit sind Bestandteil des ePortfolios.

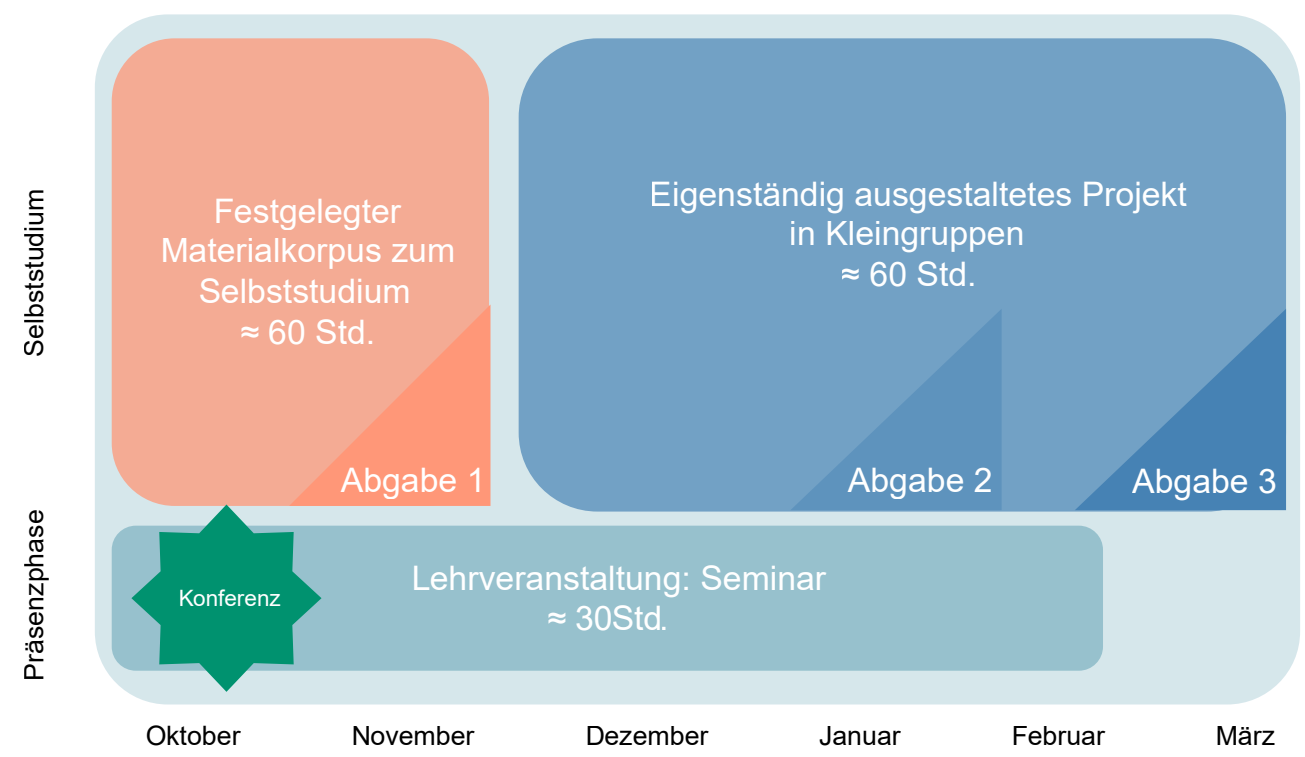

Abb. 4.: Schematische Darstellung zum Aufbau des Moduls Medienpädagogik (Eigene Darstellung).

Die Konzeption berücksichtigt Ergebnisse der Vernetzungstreffen. Mit dem Materialkorpus wird dem Wunsch begegnet, grundlegende Fähigkeiten (im Bereich Bildungsraum Schule in einer von digitalen Medien durchdrungenen Welt/Kultur und Unterricht mit digitalen Medien planen, durchführen und reflektieren) zu vermitteln und zu einer kritisch-reflexiven Haltung beizutragen (z.B. durch die Beantwortung kritischer Reflexionsfragen). Die Möglichkeit, aus unterschiedlichen Seminaren mit unterschiedlichen Schwerpunkten wählen zu können, ist an den Wunsch der Fachdidaktikerinnen 
und -didaktikern angelehnt, unterschiedliche Themen im Modul zu behandeln. Der Wunsch nach Ergänzung der theoretischen Grundlagen durch praxisbezogene Fähigkeiten soll dem Modul durch die praktische Projektarbeit gerecht werden. Dem Wunsch nach Einsatz (fach-)spezifischer Werkzeuge soll dadurch begegnet werden, dass sowohl im Seminar, als auch im Projekt mit unterschiedlichen Werkzeugen gearbeitet werden muss.

\section{Fazit und Ausblick}

Rückblickend lässt sich resümieren, dass das Verfahren der Vernetzungstreffen auf Grundlagen einer Matrix die Artikulation unterschiedlicher Perspektiven ermöglicht und inhaltliche sowie methodische Überschneidungen in der Lehre im Lehramtsstudium sichtbar macht. Somit können interdisziplinär Bezüge innerhalb der Lehre ermöglicht werden. Für eine nachhaltige Vernetzung zwischen den einzelnen Akteurinnen und Akteuren eröffneten die Treffen über die Matrix hinaus, die Möglichkeit, gemeinsame Kooperationsprojekte zwischen Fachdidaktik und Medienpädagogik anzustossen.

Im Januar 2019 begann die zweite Förderphase des im Rahmen der "Qualitätsoffensive Lehrerbildung» geförderten Projekts «MINTplus²: systematischer und vernetzter Kompetenzaufbau in der Lehrerbildung» an der Technischen Universität Darmstadt. In dieser steht vor allem die Weiterentwicklung des Vernetzungsmoduls Medienpädagogik, welches im Wintersemester 2018/2019 pilotiert wurde, und die Organisation einer jährlich stattfindenden Konferenz im Vordergrund. Die universitätsinterne Konferenz, die im Teilprojekt «Professionalisierungsschiene Digitalisierung» verankert ist, richtet sich an die Projektmitarbeiterinnen und Projektmitarbeiter, Kooperationspartnerinnen und Kooperationspartner, Studierende an der Technischen Universität Darmstadt sowie (angehende) Lehrpersonen und Interessierte. Studierende des Lehramts sollen in diesem Rahmen ihre eigenen erstellten Produkte zur Gestaltung digitalgestützter Unterrichtsprozesse (z.B. Ergebnisse des Vernetzungsmoduls Medienpädagogik) vorstellen und somit einen Beitrag zum praktischpädagogischen Diskurs leisten.

\section{Literatur}

Arbeitsgruppe Erziehungswissenschaft. 1997. "Arbeitsgruppe «Erziehungswissenschaft»». In Neue Medien - neue Aufgaben für die Lehrerausbildung: Tagungsdokumentation, herausgegeben von Gerhard Tulodziecki und Sigrid Blömeke. 39-53. Gütersloh: Verlag Bertelsmann Stiftung. 
Aßmann, Sandra, und Bardo Herzig. 2015. «Integrative Medienbildung in der Geschichtsdidaktik am Beispiel von TwHistory-Projekten». In Medien machen Geschichte. Neue Anforderungen an den geschichtsdidaktischen Medienbgegriff im digitalen Wandel, herausgegeben von C. Pallaske. 67-84. Berlin: Logos Verlag.

Blömeke, Sigrid. 2005. «Medienpädagogische Kompetenz: Theoretische Grundlagen und erste empirische Befunde». In Kompetenzdiagnostik: Theorien und Methoden zur Erfassung und Bewertung von beruflichen Kompetenzen, herausgegeben von A. Frey. 76-97. Landau in der Pfalz: Empirische Pädagogik.

BMBF, Bundesministerium für Bildung und Forschung, Hrsg. 2010. «Kompetenzen in einer digital geprägten Kultur. Medienbildung für die Persönlichkeitsentwicklung, für die gesellschaftliche Teilhabe und für die Entwicklung von Ausbildungs- und Erwerbsfähigkeit». http://www.dlr.de/pt/Portaldata/45/Resources/a_dokumente/bildungsforschung/Medienbildung_Broschuere_2010.pdf.

Bruder, Regina, und Yvonne Bachmann. 2017. «Das Projekt MINTplus im Überblick». In MINTplus - Systematischer und vernetzter Kompetenzaufbau in der Lehrerbildung, herausgegeben von R. Bruder. 6-9. Darmstadt. https://www.zfl.tu-darmstadt.de/media/zfl/projekte_ medien/projekte_mintplus/downloadbereich/Broschuere_Projekt_MINTplus_TU_Darmstadt.pdf.

Bundestag. 24.03.2015. «Deutscher: Antrag der Fraktionen der CDU/CSU und SPD: Durch Stärkung der Digitalen Bildung Medienkompetenz fördern und digitale Spaltung überwinden». http://dipbt.bundestag.de/extrakt/ba/WP18/660/66045.html.

DGfE Sektion Medienpädagogik. 2017. «Orientierungsrahmen für die Entwicklung von Curricula für medienpädagogische Studiengänge und Studienanteile». MedienPädagogik: Zeitschrift für Theorie und Praxis der Medienbildung, Nr. Theses Papers, Statements and Frameworks (Dezember), 1-7. https://doi.org/10.21240/mpaed/00/2017.12.04.X

Jörissen, Benjamin. 2011. «Medienbildung - Begriffsverständnisse und Reichweiten». MedienPädagogik: Zeitschrift für Theorie und Praxis der Medienbildung 20 (MedienbildMedienbildung im Spannungsfeld medienpädagogischer Leitbegriffe), 211-35. https://doi. $\operatorname{org} / 10.21240 / \mathrm{mpaed} / 20 / 2011.09 .20 . x$.

Kammerl, Rudolf. 2015. «Medienbildung - (k)ein Unterrichtsfach? Eine Expertise zum Stellenwert der Medienkompetenzförderung in Schulen». Hamburg: Medienanstalt Hamburg/ Schleswig-Holstein (MA HSH).

Kammerl, Rudolf, und Jönne Atzeroth. 2013. «Informationskompetenz mit modernen Schulbibliotheken fördern - Beobachtungen von Beispielen für Informationskompetenzförderung und Integration digitaler Medien im Modellprojekt «Schulbibliotheken für alle Schulen»». In Digitale Medien und Schule. Zur Rolle digitaler Medien in Schulpädagogik und Lehrerbildung, herausgegeben von Dietrich Karpa, Birgit Eickelmann und Silke Grafe. 95-109. Verlag Barbara Budrich. https://doi.org/10.2307/j.ctvdf05w3.10.

Kammerl, Rudolf, und Sandra Ostermann. 2010. «Medienbildung - (k)ein Unterrichtsfach? Eine Expertise zum Stellenwert der Medienkompetenzförderung in Schulen». Hamburg: Medienanstalt Hamburg/Schleswig-Holstein (MA HSH). 
Kleiner, Bettina, und Florian C. Klenk. 2017. «Genderkompetenzlosigkeitskompetenz: Grenzen pädagogischer Professionalisierung in der Perspektive der Queer Theory». In Jahrbuch Frauen- und Geschlechtsforschung in der Erziehungswissenschaft. Queertheoretische Perspektiven auf Bildung - Pädagogische Kritik der Heteronormativität, herausgegeben von J. Hartmann, A. Messerschmidt und C. Thon. 97-120. Opladen: Barbara Budrich.

KMK, Kultusministerkonferenz. 2012. «Medienbildung in der Schule (Beschluss der Kultusministerkonferenz vom 8. März 2012)». https://www.kmk.org/fileadmin/Dateien/veroeffentlichungen_beschluesse/2012/2012_03_08_Medienbildung.pdf.

KMK, Kultusministerkonferenz. 2017. "Strategie der Kultusministerkonferenz. Bildung in der digitalen Welt». https://www.kmk.org/fileadmin/Dateien/veroeffentlichungen_beschluesse/2018/Strategie_Bildung_in_der_digitalen_Welt_idF._vom_07.12.2017.pdf.

LKM, Länderkonferenz MedienBildung. 2015. «Kompetenzorientiertes Konzept für die schulische Medienbildung». LKM-Positionspapier. http://www.laenderkonferenz-medienbildung. de/files/Dateien_lkm/Dokumente/LKM-Positionspapier_2015.pdf.

Mayrberger, Kerstin. 2012. «Medienpädagogische Kompetenz im Wandel - Vorschlag zur Gestaltung des Übergangs in der Lehrerbildung am Beispiel mediendidaktischer Kompetenz». In Jahrbuch Medienpädagogik 9, herausgegeben von Renate Schulz-Zander, Birgit Eickelmann, Heinz Moser, Horst Niesyto und Petra Grell, 389-412. Wiesbaden: VS Verlag für Sozialwissenschaften. https://doi.org/10.1007/978-3-531-94219-3_17.

Moser, Heinz. 2010. Schule 2.0 Medienkompetenz für den Unterricht. Kronach: Link.

Moser, Heinz. 2012. «Bildungsstandards im Medienbereich». In Jahrbuch Medienpädagogik 9, herausgegeben von Renate Schulz-Zander, Birgit Eickelmann, Heinz Moser, Horst Niesyto und Petra Grell, 249-269. Wiesbaden: VS Verlag für Sozialwissenschaften. https://doi. org/10.1007/978-3-531-94219-3_12.

Niesyto, Horst. 2010. «Medienpädagogik in der Lehrerbildung in Baden Württemberg. Konzeptionelle Überlegungen und praktische Schritte zu einer medienpädagogischen Grundbildung». In Jahrbuch Medienpädagogik 9, herausgegeben von Renate Schulz-Zander, Birgit Eickelmann, Heinz Moser, Horst Niesyto und Petra Grell, 333-357. Wiesbaden: VS Verlag für Sozialwissenschaften. https://doi.org/10.1007/978-3-531-94219-3_15.

Technische Universität Darmstadt. 2017. Modulhandbuch des Studiengangs Lehramt an Gymnasien - Vernetzungsbereich. https://www.zfl.tu-darmstadt.de/media/zfl/studium_medien/studium_lag/studium_lag_studienordnungen/vernetzungsbereich/Uebergreifend_ Vernetzungsbereich_Ordnung_LaG_Modulhandbuch_Satzungsbeilage_II-2017_final.pdf.

Tulodziecki, Gerhard. 2012. «Medienpädagogische Kompetenz und Standards in der Lehrerbildung». In Jahrbuch Medienpädagogik 9, herausgegeben von Renate Schulz-Zander, Birgit Eickelmann, Heinz Moser, Horst Niesyto und Petra Grell, 271-297. Wiesbaden: VS Verlag für Sozialwissenschaften. https://doi.org/10.1007/978-3-531-94219-3_13.

Tulodziecki, Gerhard. 1997. Medien in Erziehung und Bildung: Grundlagen und Beispiele einer handlungs- und entwicklungsorientierten Medienpädagogik. 3. überarb. und erw. Aufl. Bad Heilbrunn: Klinkhardt. 